\title{
ADDICTION AND THE BRAIN: THE NEUROBIOLOGY OF COMPULSION AND ITS PERSISTENCE
}

\begin{abstract}
Steven E. Hyman ${ }^{*}$ and Robert C. Malenka
People take addictive drugs to elevate mood, but with repeated use these drugs produce serious unwanted effects, which can include tolerance to some drug effects, sensitization to others, and an adapted state - dependence - which sets the stage for withdrawal symptoms when drug use stops. The most serious consequence of repetitive drug taking, however, is addiction: a persistent state in which compulsive drug use escapes control, even when serious negative consequences ensue. Addiction is characterized by a long-lasting risk of relapse, which is often initiated by exposure to drug-related cues. Substantial progress has been made in understanding the molecular and cellular mechanisms of tolerance, dependence and withdrawal, but as yet we understand little of the neural substrates of compulsive drug use and its remarkable persistence. Here we review evidence for the possibility that compulsion and its persistence are based on a pathological usurpation of molecular mechanisms that are normally involved in memory.
\end{abstract}

The defining characteristic of addiction is compulsive, out-of-control drug use despite serious negative consequences. The life of an addicted person becomes progressively focused on obtaining, using and recovering from the effects of drugs, despite illness, disrupted relationships and failures in life roles ${ }^{1,2}$. For example, we know of a physician who, despite extensive vascular surgery and threatened amputations, continues to smoke cigarettes; many clinicians have seen individuals who attempt to smoke through a tracheotomy tube after a laryngectomy for cancer; and, despite loss of employment and life-threatening complications of cirrhosis, many people continue to drink alcohol even when drinking yields depression rather than pleasure. These examples highlight the central question of addiction: what happens in the brain to cause an addicted person to lose control of drug-taking behaviour even when experiencing serious drug-related harm?

Compulsive actions that are readily misinterpreted as willful self-destruction frustrate family members and clinicians alike, but perhaps the most problematic aspect of addiction is the high risk of relapse to drug use that persists even in abstinent addicts long after any withdrawal symptoms have abated and perhaps for a lifetime ${ }^{1-5}$. Addiction can appropriately be considered as a chronic medical illness 6 . In a 33-year follow-up study of 581 male heroin addicts admitted to a California-state drug-treatment programme between 1962 and 1964, 284 were dead by 1997, most as a result of drug overdose or violence; of the 241 subjects who could be found (mean age 57.4 years), $40.5 \%$ admitted to heroin use in the past year, $20.7 \%$ had urine that was positive for heroin at the time of the follow-up interview, and 23.5\% either refused to provide a specimen or were incarcerated ${ }^{7}$.

As is true of many chronic brain diseases, current treatments for addiction are helpful to some, but far from satisfactory. Therefore, a central goal of current research is to identify molecular mechanisms that will lead to new treatments. To achieve this goal, and to understand the pathophysiology of addiction, we must address questions that require the integration of behavioural, systems-level, cellular and molecular neuroscience. Recent reviews have addressed the complex molecular changes induced by addictive drugs in relevant 


\begin{tabular}{|c|c|}
\hline Drugs & Molecular targets relevant to addiction \\
\hline $\begin{array}{l}\text { Opioids (morphine-like drugs } \\
\text { including heroin) }\end{array}$ & $\mu$ - and $\delta$-opioid receptors (agonists) \\
\hline Barbiturates and benzodiazepines & $\begin{array}{l}\mathrm{GABA}_{\mathrm{A}} \text { receptor (enhance activation of the } \mathrm{Cl}^{-} \\
\text {channel by different mechanisms) }\end{array}$ \\
\hline $\begin{array}{l}\text { Psychomotor stimulants (cocaine } \\
\text { and amphetamines) }\end{array}$ & $\begin{array}{l}\text { Cocaine blocks dopamine, serotonin }(5-\mathrm{HT}) \text { and } \\
\text { noradrenaline transporters; amphetamines cause } \\
\text { transporter-mediated release; the dopamine } \\
\text { transporter (DAT) is the most significant target }\end{array}$ \\
\hline $\begin{array}{l}\text { Phencyclidine (PCP) and } \\
\text { related drugs }\end{array}$ & NMDA glutamate receptor channel (blocker) \\
\hline Cannabinoids & CB1 cannabinoid receptor (agonist) \\
\hline Nicotine & Nicotinic acetylcholine receptor (agonist) \\
\hline Ethyl alcohol & $\begin{array}{l}\text { GABA }_{A} \text { receptor (facilitates), NMDA glutamate } \\
\text { receptor (inhibits) and many other targets }\end{array}$ \\
\hline Inhalants & Unknown \\
\hline
\end{tabular}

neural circuits ${ }^{8}$ and the hypothesis that addictive drugs produce long-lived alterations in behaviour largely by usurping normal mechanisms of associative memory ${ }^{9}$. Here we review recent data that are consistent with this view, with the goal of providing a framework within which to study the problem of relapse and its persistence.

\section{Tolerance, dependence and withdrawal}

Addictive drugs (TABLE 1) are both rewarding (interpreted by the brain as intrinsically positive) and reinforcing (behaviours associated with such drugs tend to be repeated $)^{10}$. When these drugs are used repeatedly by vulnerable humans ${ }^{11,12}$, molecular changes in the brain ${ }^{8}$ promote continued drug taking that becomes increasingly difficult for the individual to control ${ }^{9,13,14}$. Once addiction has taken hold, it tends to follow a chronic course, in which periods of abstinence are followed by relapse to active drug use $e^{3,6}$.

In addition to producing compulsive use, the drugs listed in TABLE 1 can produce tolerance, dependence and withdrawal symptoms. Tolerance is defined as a decrease in the effect of a drug despite a constant dose, or a need for increased dosage to maintain a stable effect. Tolerance often develops to the desired pleasurable effects of cocaine, heroin and other abused drugs, leading to dosage increases that can exacerbate the molecular changes that lead to addiction. Depending on the pattern of use, some drugs, of which cocaine and amphetamine are the best studied, can also produce sensitization (enhancement) of some drug responses ${ }^{15-17}$. Tolerance to some drug effects can coexist with sensitization to others, presumably reflecting the different properties of the circuits affected.

Despite the conflation of the terms 'dependence' and 'addiction' in some clinical diagnostic systems ${ }^{18}$, they are not scientifically equivalent. Dependence, narrowly defined, refers to an adapted state of cells, circuits or organ systems that occurs in response to excessive drug stimulation. When unmasked by drug cessation, this adapted state can result in the production of cognitive, emotional or 'physical' withdrawal symptoms. Many drugs used in general medicine also produce tolerance, dependence and withdrawal without producing compulsive use (for example, $\beta$-adrenergic-agonist inhalers for asthma, $\alpha$-adrenergic-agonist nasal decongestants, and several agents used to treat hypertension or angina pectoris). So-called 'physical dependence' on drugs of abuse results from adaptations that occur in brain circuits that control directly observable bodily functions, such as heart rate or blood pressure. Ethanol, barbiturates and opiates produce physical dependence; the highly addictive drugs cocaine and amphetamine do not. Mechanisms of dependence, withdrawal and sensitization have been studied extensively using animal mod$\mathrm{els}^{16,17,19-21}$. In particular, the neural and molecular basis of physical opiate dependence and withdrawal has been thoroughly investigated as a model of drug action in the brain (reviewed in REFS 8,19,22). By contrast, convincing animal models of addiction - compulsive use despite negative consequences - are lacking ${ }^{3}$.

The desire to elevate or otherwise alter mood often motivates initial drug use. However, the pleasure (or relief of dysphoric moods) produced by drugs often habituates; for drugs such as alcohol and nicotine, pleasure can be markedly reduced over time by medical complications. Addicted individuals sometimes describe their continuing drug use as an attempt to re-experience remembered 'highs', often without success. Robinson and Berridge $^{23}$ have argued that in addiction, as opposed to drug experimentation, the dominant emotional response to drugs is no longer 'liking', but intense drug urges or 'wanting. They have proposed an incentivesensitization theory of addiction, which posits that brain systems that are normally involved in incentive motivation (but not in pleasure) become hypersensitive to drugs and drug-associated stimuli, markedly increasing the incentive salience of these stimuli $i^{13,23}$.

Just as reliable drug-induced pleasure does not explain compulsive use, neither does the desire to avoid withdrawal symptoms. If the discomfort associated with withdrawal were the major obstacle to recovery, close supervision of addicted individuals for several weeks until withdrawal symptoms diminished would yield a cure - even for drugs such as alcohol, barbiturates and opiates, which produce the most serious withdrawal syndromes. In fact, late relapse, long after withdrawal symptoms have cleared, is a fundamental problem for addicts. Moreover, for psychostimulants such as cocaine and amphetamine, physical withdrawal symptoms are absent, and emotional withdrawal symptoms are variable and can be mild. Finally, it is worth recalling that withdrawal symptoms from non-addictive drugs can also be severe at times. In summary, pleasure, tolerance, dependence and withdrawal are clinically significant, but do not explain compulsive drug use or late relapses ${ }^{3,4,13}$.

\section{Cue-mediated relapse}

Cues associated with previous drug use can initiate drug craving and conditioned emotional responses in addicts, and are associated clinically with relapse both during active drug use and after a period of abstinence ${ }^{3,24}$. 
Drug-conditioned cues can be environmental or interoceptive. For example, the risk of relapse is elevated when addicts encounter people, places or paraphernalia associated with earlier drug use. Cues can elicit drug-related behaviour in animal models $s^{4,25-27}$, including sensitized responses to psychostimulants ${ }^{28-31}$. That sensitization in animal models can be context dependent and long-lived has raised the possibility that it could be a model of relapse in humans, and it has been incorporated into the incentive-sensitization model of addiction ${ }^{13,23}$. Stress also produces resumption of drug self-administration in rodent models ${ }^{32}$; its role in relapses in human addicts seems to be significant and deserves further study.

In laboratory settings, drug-associated cues can elicit conditioned emotional responses, including drug urges, in human addicts ${ }^{33}$. Studies of responses to drugconditioned cues using positron emission tomography $(\mathrm{PET})^{34-36}$ and functional magnetic resonance imaging $(\mathrm{fMRI})^{37}$ have shown activation of prefrontal cortical regions and the amygdala, a brain region involved in the consolidation of stimulus-reward associations $s^{38}$. Some of these imaging studies have also found activation of the nucleus accumbens (NAc) and other regions. The cue-induced brain activations observed with PET and fMRI correlate with the onset of subjective drug urges and physiological responses, such as activation of the autonomic nervous system.

A debate continues as to whether cues initiate relapses through the mediation of intense urges ${ }^{23}$, which can be consciously experienced, by the activation of overlearned and unconscious behavioural repertoires ${ }^{9,39}$, or by some combination of the two. However, there is agreement that cue-initiated relapses can occur even in individuals who have strongly resolved never to use drugs again, often without the addicted person having insight into what is happening to them ${ }^{3,39}$.

\section{Neural substrates of addictive drug action}

There is strong evidence that the dopaminergic system that projects from the ventral tegmental area (VTA) of the midbrain to the NAc, and to other forebrain sites including the dorsal striatum, is the major substrate of reward and reinforcement for both natural rewards and addictive drugs ${ }^{4,40-42}$. The NAc is involved in responding to the motivational significance of stimuli, and the dorsal striatum is involved in the learning and execution of behavioural sequences that permit an efficient response to those cues. Addictive drugs increase the levels of synaptic dopamine in the NAc; pharmacological antagonist and lesion experiments have shown that dopamine is generally required for reward and reinforcement $t^{4,40,43}$. Opiates represent a partial exception to the central role of dopamine. Although opiates can produce reinforcement by dopamine release $\mathrm{e}^{44}$, they can also interact directly with opioid receptors on NAc neurons ${ }^{19}$. Under normal circumstances, this dopaminergic circuit is a crucial substrate for the rewarding and reinforcing effects of positive natural stimuli associated with survival, such as food and reproductive opportunities. Whether related to drug taking or survival, actions that increase synaptic dopamine in this brain 'reward' circuitry tend to be repeated ${ }^{10,45}$. The use of fMRI during drug infusions has begun to show that this reward circuit is also activated by addictive drugs in humans ${ }^{46}$.

Studies by Schultz and colleagues have shown that the function of dopamine in this circuit is not simply to signal reward. The midbrain dopamine neurons of monkeys show a complex and changing response to rewards as the monkey learns. An unanticipated reward produces transient firing of dopamine neurons, but as the monkey learns to recognize the signals that predict a reward (for example, a programmed flash of light or the noise of the test apparatus), the response of dopamine neurons changes. The response to the reward itself habituates and, instead, the dopamine neurons fire in response to the predictors. If a predicted reward is omitted, normal basal levels of firing are suppressed; if the reward exceeds expectation, firing is enhanced ${ }^{47-50}$. These data support the hypothesis that one function of dopamine release in the forebrain is to serve as an 'errordetection' or learning signal. The firing pattern is consistent with the idea that midbrain dopamine neurons receive highly processed information from the cerebral cortex and other regions. If the significance of specific reward predictors and appropriate motor responses to them are to be learned, dopamine released in the NAc and dorsal striatum must interact with excitatory neurotransmitters carried by projection neurons from the cerebral cortex, hippocampus and amygdala, which provide many of the main inputs to the striatum. These projections carry detailed information about external context, and internal emotional and physiological states. Drug-induced synaptic plasticity in the NAc and dorsal striatum therefore contribute to addiction by consolidating drug-wanting, drug-seeking and drug-taking behaviours.

The powerful control over behaviour exerted by addictive drugs is thought to result from the brain's inability to distinguish between the activation of reward circuitry by naturally rewarding activities, such as eating, and by the consumption of drugs ${ }^{42}$ (BOX 1). Drugs can stimulate brain reward circuitry with a strength, time course and reliability that exceeds almost any natural stimulus, powerfully consolidating responses to drug-associated stimuli?

\section{Candidate cellular mechanisms}

If dopamine release is to change the behaviour of an organism so that it responds adaptively to future cues, dopamine must contribute to alterations in neural circuits that evaluate and respond to those cues. For example, the cue-dependent learning found in the monkeys studied by Schultz ${ }^{47,49,50}$, and the phenomena of cueconditioned human drug urges and conditioned relapse, require the storage of specific patterns of information in the brain. This stored information must provide internal representations of reward-related stimuli, including both their nature and emotional valence; there must also be stored patterns of information that can give rise to efficient behavioural responses. It is important to distinguish the storage of specific information related to drug experiences from changes in 


\section{Box 1 | Dopamine and euphoria: not so simple}

Use of the term 'reward circuit' to refer to the mesolimbic dopamine system (and occasionally to its downstream connections) has long provided a useful heuristic for the role of these neurons in the powerful effects of addictive drugs. At the same time, this terminology implies that an important role for dopamine in the nucleus accumbens (NAc) or prefrontal cortex is to directly mediate the hedonic impact of drugs; that is, the subjective experience of 'pleasure'. There is substantial evidence that this is not the case and, indeed, that dopamine has more complex roles. Unpleasant, noxious stimuli, such as tail pinch in a rat, can increase the firing of midbrain dopamine cells and thereby cause the release of dopamine in the NAc, as can novel stimuli that have no obvious rewarding or pleasurable properties. As described in the main text, after an experimental animal has learned about the cues that predict reward, the timing of dopamine neuron firing, and therefore of release, is such that dopamine is released with the cues, not with consumption of the reward. This observation is more consistent with dopamine serving as an 'error' or 'learning' signal than as a mediator of hedonic experience ${ }^{47-50}$. Moreover, rats with extensive dopamine depletion in the NAc and dorsal striatum (produced by the neurotoxin 6-hydroxydopamine) have normal hedonic reaction patterns to sweet (sucrose) versus bitter tastes, and can even learn about new hedonic stimuli, indicating that dopamine might be more involved in learning the motivational significance of a stimulus than in learning new 'likes' and 'dislikes' ${ }^{112}$. Delineating the exact circuitry and neurotransmitters (a role for endogenous opioid peptides has been proposed) that do mediate 'pleasure' will require further investigation. The investigation of circuitry mediating pleasure could benefit from neuroimaging in humans, as pleasure is a subjective experience that can be defined only operationally in animals, but can be directly described by human subjects.

overall dopamine release or dopamin e responsiveness. For example, globally diminished dopamine responsiveness might develop as a homeostatic response to excessive drug stimulation. This adaptation might affect the overall responsiveness of an organism during the drug withdrawal period. Such global changes cannot explain the response to particular drug-conditioned cues. The encoding of specific information about cues and their significance cannot rely on dopamine alone, because dopamine neurons seem to fire in unison ${ }^{47,49,50}$ and to affect their targets diffusely. There is extensive evidence that dopamine interacts with cortically derived glutamate to produce changes in behaviour ${ }^{26,51-53}$ (FIG. 1).

The experience-dependent or drug-dependent reorganization of neural circuitry can occur by several general mechanisms. Changes in synaptic strength (synaptic plasticity) might result from a change in neurotransmitter release, neurotransmitter receptors or receptor-mediated signalling. Alternatively, changes in the intrinsic excitability of neurons might follow changes in the properties or numbers of voltage-dependent ion channels. A third possibility is that morphological changes, such as the generation of new synaptic connections or the pruning away of pre-existing ones, might be initiated by various forms of synaptic plasticity. The best candidate mechanisms for associative, synapse-specific plasticity are various forms of longterm potentiation (LTP) and long-term depression (LTD). These are suggested to be crucial for many forms of experience-dependent plasticity, including learning and memory $y^{54,55}$. LTP and LTD are therefore important candidate mechanisms for the drug-induced reorganization of neural circuitry that occurs during addiction. Indeed, there is abundant correlative evidence that LTP- and LTD-like changes can occur in mesolimbic dopamine structures as a consequence of drug administration, and that these are important in the development of addiction ${ }^{51,53,56}$.

Much of the work on the detailed mechanisms underlying LTP and LTD has been carried out in the hippocampus, both because of its importance in declarative memory and for technical reasons ${ }^{57,58}$. However, it is clear that virtually all excitatory synapses in the mammalian brain express LTP and LTD, although the mechanisms differ among different brain regions. In the context of addiction, it is important to study synaptic plasticity in the relevant neurons found in the midbrain, NAc and dorsal striatum.

Both LTP and LTD can be elicited at excitatory synapses in the NAc. Like forms of plasticity studied in the hippocampus, both require the activation of NMDA ( $N$-methyl-D-aspartate) glutamate receptors (NMDAR) $)^{59-61}$. Initial studies have focused primarily on the synapses made by prelimbic cortical afferents, and it remains to be determined whether other excitatory afferents to the NAc express similar forms of plasticity. In contrast to findings in the dorsal striatum (see below), the activation of dopamine receptors is not required for the generation of LTP or LTD in the NAc ${ }^{59-61}$. However, the application of amphetamine (which increases the concentration of dopamine at the synapse by inducing release) blocks the generation of $\mathrm{LTP}^{62}$, perhaps because dopamine mediates the depression of glutamate release, even during an LTP-inducing tetanus ${ }^{63}$. An intriguing feature of LTP in the NAc is that it seems to be accompanied by a decrease in the NMDAR-mediated component of synaptic responses ${ }^{60}$. The functional significance of this is not yet understood.

Very little is known about the detailed biochemical and molecular mechanisms that underlie LTP and LTD in the NAc. By contrast, a great deal is known about the mechanisms of synaptic plasticity, particularly LTD, in the dorsal striatum. LTD in this area does not require NMDAR activation, but instead requires a rise in $\mathrm{Ca}^{2+}$ that is mediated by the activation of voltage-dependent $\mathrm{Ca}^{2+}$ channels ${ }^{64}$. It also requires the activation of both D1like and D2-like dopamine receptors by endogenously released dopamine, and involves a complex cascade of intracellular signals that include several protein kinas$\mathrm{es}^{64-66}$. On the other hand, LTP in the dorsal striatum requires NMDAR activation as well as the activation of D1-like receptors; moreover, it is enhanced by the inhibition of D2-like receptors ${ }^{64}$. (In the striatum, D1 receptors are coupled to $\mathrm{G}_{\mathrm{s}} / \mathrm{G}_{\mathrm{olf}}$, stimulating adenylyl cyclase to produce the intracellular second messenger cyclic AMP, which in turn activates the cAMP-dependent protein kinase (PKA); D2 receptors are coupled to $G_{i} / G_{o}$, and so tend to inhibit adenylyl cyclase and to activate an inwardly rectifying $\mathrm{K}^{+}$current.) So, the forms of synaptic plasticity in the dorsal striatum seem to differ markedly from those observed in the NAc. Indeed, even the basic synaptic actions of dopamine differ in the two structures ${ }^{63}$.

The study of synaptic plasticity in midbrain dopamine regions (the VTA and substantia nigra pars compacta) is in its infancy. LTP at excitatory synapses on midbrain dopamine neurons is NMDAR dependent, but for reasons that are unclear, it is often difficult to generate. 


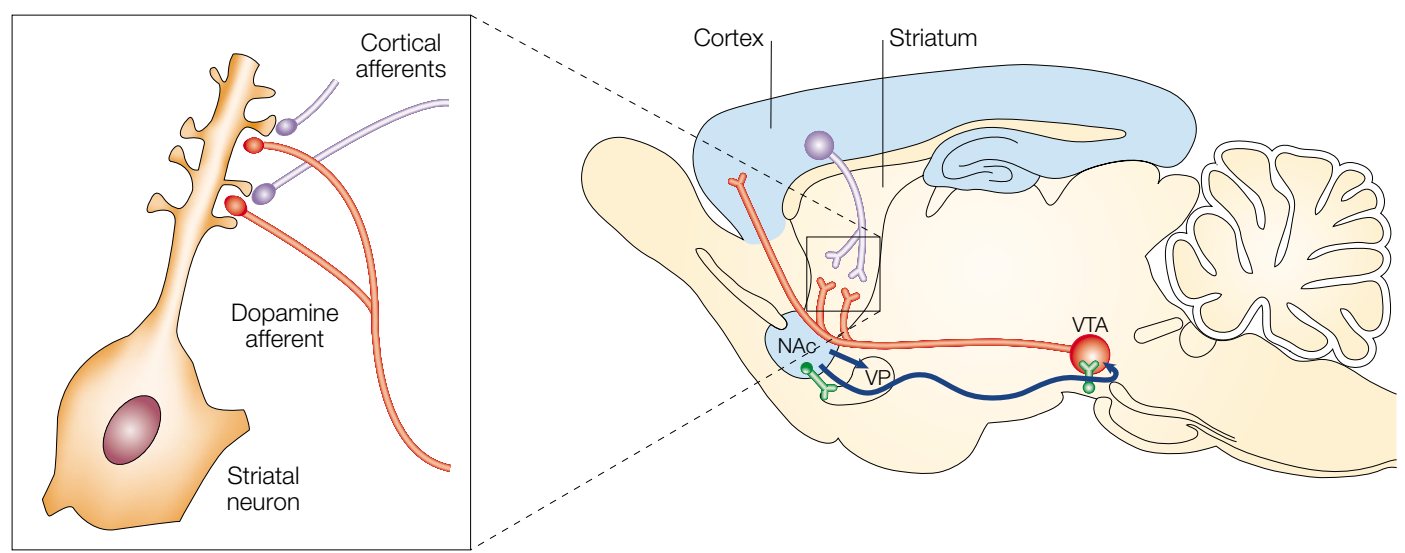

Figure 1 | Dopamine-glutamate interactions in the striatum. Approximately 95\% of neurons in the dorsal striatum and nucleus accumbens (NAc) are medium-sized spiny projection neurons, which use GABA ( $\gamma$-aminobutyric acid) as their main neurotransmitter. These neurons receive glutamatergic projections from the cerebral cortex, which form well-defined synapses on the heads of dendritic spines. Dopaminergic axons from the midbrain pass by the necks of spines, where they release neurotransmitter; however, dopamine receptors are widely distributed on the cell membrane, including the soma. The figure shows the principal brain regions discussed in the main text. VP, ventral pallidum; VTA, ventral tegmental area.

GABA ( $\gamma$-aminobutyric acid)-releasing cells in the VTA do not express LTP ${ }^{67}$. LTD is generated by the activation of voltage-dependent $\mathrm{Ca}^{2+}$ channels and does not require the activation of dopamine receptors ${ }^{61,68}$. However, it is inhibited or blocked by dopamine and amphetamine through the activation of D2-like receptors ${ }^{61,68}$, a modulation with potentially important functional implications (see below). Recent work has begun to explore the intracellular mechanisms that mediate LTD in the VTA, which seem to involve increases in cAMP and the activation of PKA - a mechanism quite distinct from other forms of LTD that have been studied ${ }^{69}$.

The demonstration of synaptic plasticity at excitatory synapses in mesolimbic dopaminergic structures, as well as the correlative evidence for changes in glutamate receptor expression ${ }^{8}$ and single-unit responses to glutamate after in vivo exposure to drugs of abuse, support a role for synaptic plasticity in the development of addiction. However, the crucial question remains as to whether drugs of abuse actually elicit changes in synaptic strength in vivo. To address this issue, Ungless et al. ${ }^{70}$ prepared midbrain slices from animals that had received a single injection of cocaine one day earlier, and assayed synaptic strength using whole-cell recording techniques. The single in vivo exposure to cocaine caused a marked potentiation of synaptic strength that was due to an upregulation of the number or function (or both) of synaptic AMPA ( $\alpha$-amino-3-hydroxy-5-methyl-4isoxazole propionic acid) glutamate receptors (AMPAR) and shared mechanisms with the LTP induced in slices. The cocaine-induced potentiation was detectable 5 but not 10 days after exposure to cocaine; like LTP, it did not occur in GABA-containing cells, and was prevented in vivo by the co-administration of an NMDAR antagonist. The mechanisms by which cocaine causes the change in synaptic weights are unknown, but one contributing factor might be the dopamine-mediated inhibition of LTD mentioned above ${ }^{61,68}$. When applied to a slice preparation of the VTA, nicotine also elicits LTP in dopaminergic cells when paired with postsynaptic depolarization $^{71}$. So, it will be interesting to learn whether the in vivo administration of nicotine and other drugs of abuse causes similar changes to those observed with cocaine. The idea that such drug-induced synaptic plasticity can contribute significantly to aspects of addiction is bolstered by the recent finding that stimulation of glutamatergic synaptic transmission in the VTA leads to a relapse in cocaine-seeking behaviour ${ }^{72}$, although the source of such stimulation under more natural conditions remains unclear ${ }^{73}$.

A similar study has been carried out in the NAc, slices of which were prepared 10-14 days after prolonged (5-day) in vivo administration of cocaine, which caused behavioural sensitization ${ }^{74}$ (M. J. Thomas et al., unpublished observations). Cells in the shell but not the core of NAc slices prepared from the cocaine-treated animals showed a decrease in strength at excitatory synapses made by prefrontal cortical afferents. LTD was also diminished, indicating that the decrease was due to mechanisms shared with LTD. As is the case for changes in the VTA, the mechanisms responsible for this druginduced synaptic plasticity in the NAc are unclear. The acute administration of amphetamine to slices blocks LTP in the NAc, and this effect disappears in slices prepared from animals that have been chronically exposed to amphetamine ${ }^{62}$. If this also occurs after in vivo cocaine exposure, such an action could initially enhance the likelihood of generating LTD.

It has long been suggested that long-term memories are associated with the formation of new synaptic connections that lead to altered circuit function. Morphological changes associated with synaptic plasticity have been demonstrated in the marine sea slug Aplysia ${ }^{75}$ and in rodents. For example, localized formation of new spine-like structures has been observed in association with a later phase of hippocampal $\mathrm{LTP}^{76}$. In classically conditioned rabbits, synaptogenesis, limited to newly formed multiple-synapse boutons, occurred in the CA1 


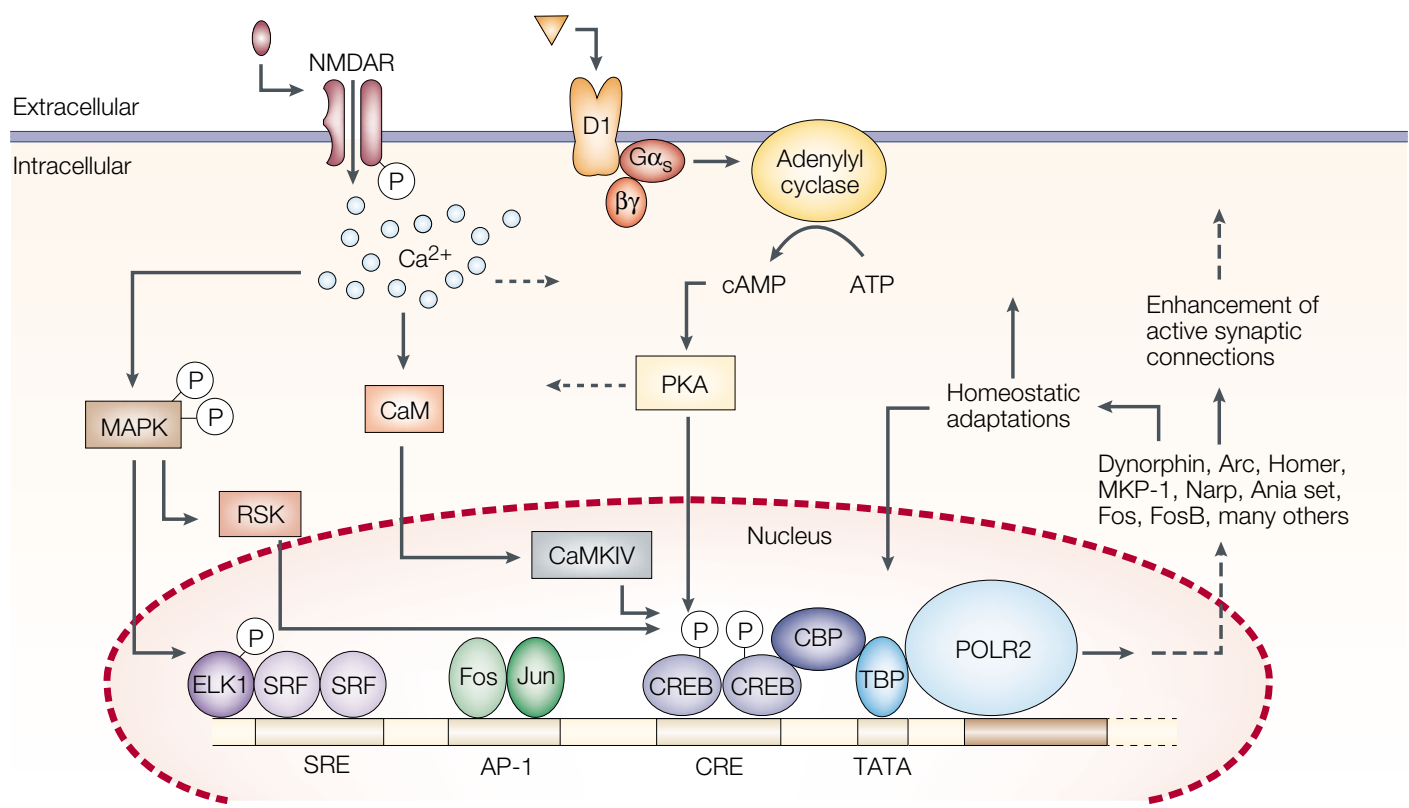

Figure 2 | Signalling to the nucleus stimulated by dopamine and glutamate. A D1 dopamine receptor and NMDA ( $N$-methylD-aspartate) glutamate receptor (NMDAR) are shown, as might be co-expressed on a medium spiny neuron. D1 stimulation activates the cyclic AMP cascade (see text) and NMDAR stimulation permits $\mathrm{Ca}^{2+}$ entry. These second messengers activate multiple kinases, including cAMP-dependent protein kinase (PKA) and calcium/calmodulin-dependent protein kinase type IV (CaMKIV), and the mitogen-activated protein kinase (MAPK)-ribosomal protein S6 kinase (RSK) pathways, which converge on the transcription factor CREB (cAMP response element (CRE)-binding protein, shown binding a CRE on the DNA). Other elements shown are a serum response element (SRE) and the AP-1 binding site, and the TATA element, which binds general transcription factors and RNA polymerase II (POLR2). The figure illustrates the hypothesis that CREB is a key regulator of genes involved in homeostatic neuroadaptations, such as dynorphin, and also of genes that might have a role in synaptic remodelling. Clearly, however, other transcription factors must also be involved in both processes. CaM, calmodulin; CBP, CREB-binding protein; ELK1, member of the ETS family of transcription factors; MKP-1, dual-specificity MAPK phosphatase; SRF, serum response factor; TBP, TATA-box-binding protein. Adapted with permission from REF. 9 @ 2000 Elsevier Science.

ASYMMETRICAL SYNAPSES Synaptic contacts in which the postsynaptic thickening is wider than the presynaptic one. They are thought to comprise largely excitatory connections. Symmetrical synapses, in contrast, are characterized by pre- and postsynaptic thickenings of roughly similar widths and are thought to be inhibitory.

CONDITIONED PLACE PREFERENCE

The development in an experimental animal of a preference for a location that is repeatedly paired with a rewarding stimulus (for example, cocaine) region of the hippocampus ${ }^{77}$. Structural modifications to dendritic structures have also been sought after alterations in dopaminergic inputs to the striatum and NAc. Dopamine denervation reduces dendritic spine density ${ }^{78-80}$ and the number of ASYMMETRICAL SYNAPSES in the striatum ${ }^{81}$. By contrast, repeated treatments with amphetamine or cocaine increase dendritic spine density and the number of branched spines in the NAc and in prefrontal cortex. These alterations last for at least 4 weeks after drug exposure ends ${ }^{82,83}$. However, demonstration of the effect of drug-induced alterations in spine morphology on altered synaptic number and function, and ultimately on altered behaviour such as context-dependent sensitization, remains an important challenge.

Clearly, the work reviewed here represents the early stages of investigation into the mechanisms of synaptic plasticity in the NAc, dorsal striatum and other brain regions associated with addiction. As mechanisms of synaptic plasticity are identified in reduced preparations, it will be important to examine their role in intact, behaving animals. However, it is already apparent that, like other forms of experience-dependent plasticity that have been studied, persistent druginduced behavioural changes probably occur because of their ability to elicit long-lasting changes in synaptic weights in crucial brain circuits.

\section{Candidate molecular mechanisms}

Because addiction is a long-lasting if not permanent state, the focus of many recent molecular investigations has been on mechanisms that could be responsible for this persistence. At the extremes of time course, two types of molecular change could be responsible for the persistence of addiction: long-lived or permanent up- or downregulation of the expression of crucial molecular species; or a brief burst of gene expression or protein translation that confers long-term alterations on behaviour by causing the physical remodelling of synapses and circuits. Relatively long-lasting, but not permanent, drug-induced changes in the expression levels of messenger RNA species and proteins have been documented ${ }^{84}$, as has the transient drug-regulated expression of many genes $^{85}$. Many molecular changes have been correlated with alterations in behavioural measures related to drug use, such as CONDITIONED PLACE PREFERENCE or sensitization, but demonstrating causal relationships in behaving animals has been difficult ${ }^{8}$. The pieces of the puzzle have not yet fallen into place to give a satisfactory picture of the molecular underpinnings of synaptic plasticity in the VTA, NAc and dorsal striatum, let alone in addiction. The task will be complex because, rather than a single lynchpin molecule, there are likely to be complicated cascades of events in multiple cells and circuits. Because the panoply of molecular adaptations to drugs of abuse has 
recently been reviewed ${ }^{8,86}$, we will discuss only a few illustrative examples.

Perhaps the longest-lived molecular alterations yet found to be induced by drugs of abuse (but also by other perturbations of dopamine signalling) are stable, post-translationally modified forms of the $\Delta$ FosB pro$\operatorname{tein}^{84}$. The acute administration of cocaine, opioids or nicotine causes the transient induction of several members of the Fos family of transcription factors, which couple with Jun proteins in the AP-1 complex of transcription factors. However, with repeated treatment with cocaine or other drugs, IMMEDIATE-EARLY GENES (IEGs) become refractory to further induction and stable isoforms of $\Delta \mathrm{FosB}$ are expressed. Transgenic mice that are engineered to overexpress $\Delta \mathrm{FosB}$ in the dorsal striatum and NAc, under the control of a tetracycline-responsive element, show increased conditioned place preference to cocaine, and increased expression of the AMPAR subunit GluR $2^{87}$. Levels of $\triangle$ FosB can be increased by drugs of abuse for up to 4 weeks, but because they return to basal levels they cannot, by themselves, explain late relapses. However, because $\Delta$ FosB is a transcription factor, its downstream effects could far outlast its expression. Mice overexpressing $\Delta \mathrm{FosB}$ show upregulated expression of the cyclin-dependent kinase Cdk5, which has been implicated in the remodelling of neural processes $^{88}$. As such, Cdk5 is an attractive candidate for involvement in the remodelling of neuronal circuits in response to addictive drugs.

The search for prolonged but ultimately reversible up- or downregulation of particular molecules has been fruitful in the investigation of tolerance, dependence and withdrawal. But if addiction is based on mechanisms of memory similar to those that have been investigated in invertebrate models, such as Aplysia and Drosophila, and in the mammalian hippocampus, a different search strategy would be more appropriate. Although there is a long way to go in understanding memory, its persistence does not seem to depend on increased levels of one or more proteins, but rather on altered patterns of synaptic connectivity. Relevant alterations in protein phosphorylation, gene expression and protein levels are generally thought to be transient, and to create permanence by remodelling synapses and circuits. Indeed, the long-term upregulation of crucial molecules might occlude mechanisms needed to reorganize or add to existing memories.

Interestingly, one transcription factor, the cAMPresponse-element-binding protein (CREB), is involved in the expression of genes that show prolonged upregulation in response to stimulation by addictive drugs, and also in the expression of genes that are induced rapidly and transiently, including IEGs such as Fos (FIG. 2). CREB is activated by multiple protein kinases, including PKA, and several $\mathrm{Ca}^{2+}$-dependent protein kinases $^{89}$, including calcium/calmodulin-dependent protein kinase type IV (CaMKIV), which is found in the nucleus of many neurons. Because CREB is a 'coincidence detector', in that it can be conjointly regulated by both the cAMP and $\mathrm{Ca}^{2+}$ pathways, it has long been a candidate for involvement in processes related to LTP and long-term memory. CREB is involved in synaptic plasticity, including late-phase LTP, and behaviours requiring long-term memory, with different pieces of the puzzle being investigated in Aplysia, Drosophila and mouse ${ }^{90-95}$.

In the striatum, psychostimulants such as amphetamine produce phosphorylation of CREB on serine 133 through stimulation of dopamine D1 receptors and the cAMP cascade ${ }^{96}$. However, there is evidence that significant levels of CREB phosphorylation in the striatum can be achieved only when both the cAMP and $\mathrm{Ca}^{2+}$ pathways are activated. For example, in primary cultures of embryonic striatal neurons, D1 agonists cause phosphorylation of CREB and IEG expression, but this is blocked by NMDAR antagonists or removal of $\mathrm{Ca}^{2+}$ from the medium ${ }^{96,97}$.

In rodent models, prolonged activation of D1 receptors or administration of drugs that increase synaptic dopamine, such as cocaine and amphetamine, lead to increased expression of several gene products, including dynorphin, an endogenous opioid peptide that is encoded by a CREB-regulated gen ${ }^{98}$. Dynorphin precursor mRNA has also been found to be elevated in the dorsal striatum and NAc of human cocaine abusers that are studied post mortem ${ }^{99}$. Dynorphin activates $\kappa$-opioid receptors on presynaptic dopamine terminals, causing decreased dopamine release ${ }^{100}$. So, increases in dynorphin expression could be one of the many compensatory mechanisms that would blunt excess drug-induced dopamine stimulation ${ }^{101}$. Moreover, $\mathrm{K}$-receptor agonists are aversive in both humans and rats ${ }^{102}$, so an increase in dynorphin expression resulting from cocaine or amphetamine administration could contribute to DYSPHORIA during withdrawal ${ }^{103}$. The overexpression of CREB in the ventral striatum, mediated by a CREB-expressing viral vector, also increases dynorphin expression, and reduces the rewarding effects of cocaine ${ }^{104}$. The increase in striatal dynorphin mRNA levels is relatively longlasting ${ }^{85,105}$, but diminishes over several days when drug administration ceases. On the basis of these observations, the upregulation of striatal dynorphin by cocaine and amphetamine has been proposed to contribute to tolerance, dependence and withdrawal symptoms by opposing dopamine release. Because neuropeptides are thought to diffuse over long distances in the brain, and because the upregulation of prodynorphin mRNA occurs throughout the striatum and NAc, dynorphin is not a good candidate for involvement in synapsespecific information storage. So, in the search for molecules that might be important in late cue-dependent relapses, other molecular candidates, including other CREB-regulated genes, have been sought.

On the basis of the hypothesis that transiently expressed genes might initiate persistent changes in synaptic structure, several groups have initiated screens of dopamine-regulated genes in relevant brain regions. Dopamine D1 receptor agonists, cocaine and amphetamine induce many genes in striatal neurons ${ }^{85,98,106}$. Most of the mRNAs induced by these treatments are transiently expressed and return to baseline levels of expression within a few hours to a day ${ }^{85,107}$. Interestingly, these 
genes overlap substanstially with those that are induced in association with hippocampal LTP, including Homer, Narp (neuronal-activity-regulated pentraxin) and Arc (activity-regulated cytoskeleton-associated protein) which might be involved in the regulation of synaptic function ${ }^{108-111}$

\section{Concluding remarks}

Clearly, the task of relating genes that are induced by addictive drugs to synaptic plasticity and synaptic remodelling, and relating synaptic plasticity to relevant behaviours, is in its early stages. One central part of the effort will be the development of improved models of compulsive drug use despite negative consequences. Although drug self-administration by rodents has provided important information, it is difficult to argue that it truly models compulsion, when the alternative to self-administration is solitude in a shoebox cage. Despite the challenges in terms of physiology and behaviour, it would be ideal if models could be devel- oped in the mouse, because of its status as a genetic model. The ability to make transgenic animals with increasing spatio-temporal control of transgene expression, combined with the use of gene microarrays $^{88}$ and, ultimately, proteomics tools to study the downstream consequences of gene expression, should permit substantial inroads into a difficult but clinically important set of problems.

Here we have described recent evidence for the concept $^{9}$ that the central behavioural features of addiction result from the ability of drugs to usurp normal mechanisms of memory in crucial survival circuits. The persistence of addiction, with its striking phenomenon of cuedependent relapse risk, is proposed to result from molecular processes that ultimately remodel synapses and circuits. We believe that more rapid progress will be made in the study of addiction if it increasingly proceeds together with, rather than in isolation from, other areas of neuroscience that are focused on understanding the mechanisms of learning and memory.
1. Hyman, S. E. A man with alcoholism and HIV infection. J. Am. Med. Assoc. 274, 837-843 (1995).

2. Hyman, S. E. Clinical crossroads. A 28 year old man addicted to cocaine. J. Am. Med. Assoc. (in the press).

3. O'Brien, C. P., Childress, A. R., Ehrman, R. \& Robbins, S. J. Conditioning factors in drug abuse: can they explain
compulsion? J. Psychopharmacol. 12, 15-22 (1998). A clear summary of the clinical correlates of classical

4. Wise, R. A. \& Bozarth, M. A. A psychomotor stimulant theory of addiction. Psychol. Rev. 94, 469-492 (1987). A seminal and classic conceptualization of addiction Wise, R. A. Addiction becomes a brain disease. Neuron $\mathbf{2 6}$ 27-33 (2000).

6. McLellan, A. T., Lewis, D. C., O'Brien, C. P. \& Kleber, H. D. Drug dependence, a chronic medical illness: implications for treatment, insurance, and outcome evaluation. J. Am. Med. Assoc. 284, 1689-1695 (2000).

7. Hser, Y. I., Hoffman, V., Grella, C. E. \& Anglin, M. D. A 33 year follow-up of narcotics addicts. Arch. Gen. Psychiatry 58, 503-508 (2001).

8. Nestler, E. J. Molecular basis of long-term plasticity underlying addiction. Nature Rev. Neurosci. 2, 119-128 (2001). The most up-to-date review of drug-induced molecular changes in the brain

9. Berke, J. D. \& Hyman, S. E. Addiction, dopamine, and the molecular mechanisms of memory. Neuron 25, 515-532 (2000)

Focuses on mechanisms that can explain the compulsive use of psychostimulants (cocaine and amphetamine) and late relapse. The proposed centra role of associative learning mechanisms forms a role of associative learning

10. White, N. M. Reward or reinforcement: what's the difference? Neurosci. Biobehav. Rev. 13, 181-186 (1989)

11. Kendler, K. S., Karkowski, L. M., Neale, M. C. \& Prescott, C. A. Illicit psychoactive substance use, heavy use, abuse, and dependence in a US population-based sample of male twins. Arch. Gen. Psychiatry 57, 261-269 (2000).

12. Tsuang, M. T. et al. Genetic influences on DSM-III-R drug abuse and dependence: a study of 3,372 twin pairs. Am. J. Med. Genet. 67, 473-477 (1996).

13. Robinson, T. E. \& Berridge, K. C. The psychology and neurobiology of addiction: an incentive-sensitization view. Addiction 95, S91-117 (2000).

14. Di Chiara, G. A motivational learning hypothesis of the role of mesolimbic dopamine in compulsive drug use. J. Psychopharmacol. 12, 54-67 (1998).

15. Kalivas, P. W. \& Stewart, J. Dopamine transmission in the initiation and expression of drug- and stress-induced sensitization of motor activity. Brain Res. Brain Res. Rev. 16 223-244 (1991).

16. Anagnostaras, S. G. \& Robinson, T. E. Sensitization to the psychomotor stimulant effects of amphetamine: modulation psychomotor stimulant effects of amphetamine: modulation
by associative learning. Behav. Neurosci. 110, 1397-1414 (1996).
17. Badiani, A., Anagnostaras, S. G. \& Robinson, T. E. The development of sensitization to the psychomotor stimulant effects of amphetamine is enhanced in a novel environment. Psychopharmacology (Berl.) 117, 443-452 (1995).

18. American Psychiatric Association. Diagnostic and Statistical Manual of Mental Disorders (American Psychiatric Press, Washington DC, 1994).

19. Nestler, E. J. Under siege: the brain on opiates. Neuron $\mathbf{1 6}$ 897-900 (1996).

20. Markou, A. \& Koob, G. F. Postcocaine anhedonia. An animal model of cocaine withdrawal. Neuropsychopharmacology 4, 17-26 (1991)

21. Weiss, F., Markou, A., Lorang, M. T. \& Koob, G. F. Basa extracellular dopamine levels in the nucleus accumbens are decreased during cocaine withdrawal after unlimited-access self-administration. Brain Res. 593, 314-318 (1992).

22. Williams, J. T. Christie, M. J. \& Manzoni, O. Cellular and synaptic adaptations mediating opioid dependence. Physiol. Rev 81, 299-343 (2001).

23. Robinson, T. E. \& Berridge, K. C. The neural basis of drug craving: an incentive-sensitization theory of addiction. Brain Res. Brain Res. Rev. 18, 247-291 (1993). Sets out a model of addiction in which drugs increas the sensitivity of circuits involved in 'wanting' rather than 'liking' drugs.

24. O'Brien, C. P., Childress, A. R., McLellan, A. T. \& Ehrman, R. Classical conditioning in drug-dependent humans. Ann. NY Acad. Sci. 654, 400-415 (1992)

25. Wikler, A. \& Pescor, F. T. Classical conditioning of a morphine abstinence phenomenon, reinforcement of opioiddrinking behavior and "relapse" in morphine-addicted rats. Psychopharmacologia 10, 255-284 (1967).

26. Kelley, A. E., Smith-Roe, S. L. \& Holahan, M. R. Response reinforcement learning is dependent on $N$-methyl-- aspartate receptor activation in the nucleus accumben core. Proc. Natl Acad. Sci. USA 94, 12174-12179 (1997).

27. Ciccocioppo, R., Sanna, P. P. \& Weiss, F. Cocaine-predictive stimulus induces drug-seeking behavior and neural activation in limbic brain regions after multiple months of abstinence: reversal by $\mathrm{D} 1$ antagonists. Proc. Natt Acad. Sci. USA 98, 1976-1981 (2001).

28. Stewart, J, De Wit, H \& Eikelboom, R. Role of unconditioned and conditioned drug effects in the selfadministration of opiates and stimulants. Psychol. Rev. $\mathbf{9 1}$ 251-268 (1984).

29. Stewart, J. Neurobiology of conditioning to drugs of abuse. Ann. NY Acad. Sci. 654, 335-346 (1992).

30. Robinson, T. E., Becker, J. B. \& Presty, S. K. Long-term facilitation of amphetamine-induced rotational behavior and striatal dopamine release produced by a single exposure to amphetamine: sex differences. Brain Res. 253, 231-241 (1982).

31. Robinson, T. E. \& Becker, J. B. Enduring changes in brain and behavior produced by chronic amphetamine administration: a review and evaluation of animal models of amphetamine psychosis. Brain Res. 396, 157-198 (1986).
32. Piazza, P. V. \& Le Moal, M. L. Pathophysiological basis of vulnerability to drug abuse: role of an interaction between stress, glucocorticoids, and dopaminergic neurons. Annu. Rev. Pharmacol. Toxicol. 36, 359-378 (1996). An exposition of the role of stress and stress hormones in drug abuse; complementary to the content of this review.

33. Ehrman, R. N., Robbins, S. J. Childress, A. R. \& O'Brien, C. P. Conditioned responses to cocaine-related stimuli in cocaine abuse patients. Psychopharmacology (Berl.) 107, cocaine abuse pa
523-529 (1992).

34. Grant, S. et al. Activation of memory circuits during cueelicited cocaine craving. Proc. Natl Acad. Sci. USA 93 12040-12045 (1996).

35. Childress, A. R. et al. Limbic activation during cue-induced cocaine craving. Am. J. Psychiatry 156, 11-18 (1999).

36. Kilts, C. D. et al. Neural activity related to drug craving in cocaine addiction. Arch. Gen. Psychiatry 58, 334-341 (2001).

37. Maas, L. C. et al. Functional magnetic resonance imaging of human brain activation during cue-induced cocaine craving Am. J. Psychiatry 155, 124-126 (1998).

38. Everitt, B. J., Morris, K. A., O'Brien, A. \& Robbins, T. W. The basolateral amygdala-ventral striatal system and conditioned place preference: further evidence of limbic-striatal interactions underlying reward-related processes. Neuroscience 42, 1-18 (1991).

39. Tiffany, S. T. A cognitive model of drug urges and drug-use behavior: role of automatic and nonautomatic processes. Psychol. Rev. 97, 147-168 (1990).

40. Di Chiara, G. \& Imperato, A. Drugs abused by humans preferentially increase synaptic dopamine concentrations in the mesolimbic system of freely moving rats. Proc. Nat/ Acad. Sci. USA 85, 5274-5278 (1988).

41. Wise, R. A. Addictive drugs and brain stimulation reward. Annu. Rev. Neurosci. 19, 319-340 (1996).

42. Robbins, T. W. \& Everitt, B. J. Neurobehavioural mechanisms of reward and motivation. Curr. Opin. Neurobiol. 6, 228-236 (1996).

43. Koob, G. F. \& Bloom, F. E. Cellular and molecular mechanisms of drug dependence. Science $\mathbf{2 4 2}, 715-723$ (1988).

44. Johnson, S. W. \& North, R. A. Opioids excite dopamine neurons by hyperpolarization of local interneurons. neurons by hyperpolarization of local

45. White, N. M. Addictive drugs as reinforcers: multiple partial actions on memory systems. Addiction 91, 921-949 (1996).

46. Breiter, H. C. et al. Acute effects of cocaine on human brain activity and emotion. Neuron 19, 591-611 (1997). The first study convincingly to show activation of brain reward circuitry in humans by cocaine.

47. Schultz, W., Apicella, P. \& Ljungberg, T. Responses of monkey dopamine neurons to reward and conditioned stimuli during successive steps of learning a delayed response task. J. Neurosci. 13, 900-913 (1993).

48. Waelti, P., Dickinson, A. \& Schultz, W. Dopamine responses comply with basic assumptions of formal learning theory Nature 412, 43-48 (2001) 
The most recent of a series of papers by Schultz and colleagues, arguing that dopamine serves as learning signal.

49. Schultz, W., Dayan, P. \& Montague, P. R. A neural substrate of prediction and reward. Science 275, 1593-1599 (1997).

50. Schultz, W. Predictive reward signal of dopamine neurons. J. Neurophysiol. 80, 1-27 (1998).

51. Kalivas, P. W. Interactions between dopamine and excitator amino acids in behavioral sensitization to psychostimulants. Drug Alcohol Depend. 37, 95-100 (1995).

52. $\mathrm{Hu}, \mathrm{X}$. T. \& White, F. J. Dopamine enhances glutamateinduced excitation of rat striatal neurons by cooperative activation of D1 and D2 class receptors. Neurosci. Lett. 224, 61-65 (1997).

53. Wolf, M. E. The role of excitatory amino acids in behavioral sensitization to psychomotor stimulants. Prog. Neurobiol. 54, 679-720 (1998)

54. Martin, S. J., Grimwood, P. D. \& Morris, R. G. Synaptic plasticity and memory: an evaluation of the hypothesis. Annu. Rev. Neurosci. 23, 649-711 (2000).

55. Bear, M. F. Progress in understanding NMDA-receptordependent synaptic plasticity in the visual cortex. J. Physio. (Paris) 90, 223-227 (1996).

56. Clark, D. \& Overton, P. G. Alterations in excitatory amino acid-mediated regulation of midbrain dopaminergic neuron induced by chronic psychostimulant administration and stress: relevance to behavioral sensitization and drug addiction. Addict. Biol. 3, 109-135 (1998).

57. Malenka, R. C. Synaptic plasticity in the hippocampus: LTP and LTD. Cell 78, 535-538 (1994)

58. Malenka, R. C. \& Nicoll, R. A. Long-term potentiation - a decade of progress? Science 285, 1870-1874 (1999). An up-to-date review of current thinking about LTP in the hippocampus.

59. Pennartz, C. M., Ameerun, R. F., Groenewegen, H. J. \& Lopes da Silva, F. H. Synaptic plasticity in an in vitro slice preparation of the rat nucleus accumbens. Eur. J. Neurosci. 5, 107-117 (1993).

60. Kombian, S. B. \& Malenka, R. C. Simultaneous LTP of nonNMDA- and LTD of NMDA-receptor-mediated responses in the nucleus accumbens. Nature $368,242-246$ (1994).

61. Thomas, M. J., Malenka, R. C. \& Bonci, A. Modulation of long-term depression by dopamine in the mesolimbic system. J. Neurosci. 20, 5581-5586 (2000).

62. Li, Y. \& Kauer, J. A. Amphetamine interferes with long-term potentiation in the nucleus accumbens. Soc. Neurosci. Abstr. 26, 1398 (2000).

63. Nicola, S. M., Surmeier, J. \& Malenka, R. C. Dopaminergic modulation of neuronal excitability in the striatum and nucleus accumbens. Annu. Rev. Neurosci. 23, 185-215 (2000). Reviews the actions of dopamine on neuronal excitability and synaptic transmission in the striatum

64. Calabresi, P., Centonze, D. \& Bernardi, G. Electrophysiology of dopamine in normal and denervated striatal neurons. Trends Neurosci. 23, S57-63 (2000).

65. Calabresi, P., Pisani, A., Mercuri, N. B. \& Bernardi, G. The corticostriatal projection: from synaptic plasticity to dysfunctions of the basal ganglia. Trends Neurosci. 19 19-24 (1996).

66. Choi, S. \& Lovinger, D. M. Decreased probability of neurotransmitter release underlies striatal long-term depression and postnatal development of corticostriatal synapses. Proc. Natl Acad. Sci. USA 94, 2665-2670 (1997)

67. Bonci, A. \& Malenka, R. C. Properties and plasticity of excitatory synapses on dopaminergic and GABAergic cells in the ventral tegmental area. J. Neurosci. 19, 3723-3730 (1999).

68. Jones, S., Kornblum, J. L. \& Kauer, J. A. Amphetamine blocks long-term synaptic depression in the ventral blocks long-term synaptic depression in the ventra These two papers describe the basic properties of These two papers describe

69. Kornblum, J. L. \& Kauer, J. A. Long-term depression (LTD) in the ventral tegmental area (VTA) requires cyclic AMP dependent protein kinase (PKA). Soc. Neurosci. Abstr. (in the press)

70. Ungless, M. A., Whisler, J. L., Malenka, R. C. \& Bonci, A. Single cocaine exposure in vivo induces long-term potentiation in dopamine neurons. Nature 411, 583-587 (2001).

This paper demonstrates that in vivo cocaine administration causes LTP at excitatory synapses in the VTA.

71. Mansvelder, H. D. \& McGehee, D. S. Long-term potentiation of excitatory inputs to brain reward areas by nicotine. Neuron 27, 349-357 (2000).
72. Vorel, S. R., Liu, X., Hayes, R. J., Spector, J. A. \& Gardner, E. L. Relapse to cocaine-seeking after hippocampal theta burst stimulation. Science 292 1175-1178 (2001)

73. Legault, M., Rompre, P. P. \& Wise, R. A. Chemica stimulation of the ventral hippocampus elevates nucleus accumbens dopamine by activating dopaminergic neuron of the ventral tegmental area. J. Neurosci. 20, 1635-1642 (2000).

74. Thomas, M. J. \& Malenka, R. C. Behavioral sensitization to cocaine is associated with changes in nucleus accumbens synaptic transmission. Soc. Neurosci. Abstr. 26, 791 (2000).

75. Bailey, C. H. \& Kandel, E. R. Structural changes accompanying memory storage. Annu. Rev. Physiol. 55 397-426 (1993)

76. Engert, F. \& Bonhoeffer, T. Dendritic spine changes associated with hippocampal long-term synaptic plasticity. Nature 399, 66-70 (1999).

77. Geinisman, Y., Berry, R. W., Disterhoft, J. F., Power, J. M. \& Van der Zee, E. A. Associative learning elicits the formation of multiple-synapse boutons. J. Neurosci. 21, 5568-5573 (2001).

78. Ingham, C. A., Hood, S. H. \& Arbuthnott, G. W. Spine density on neostriatal neurones changes with 6 hydroxydopamine lesions and with age. Brain Res. $\mathbf{5 0 3}$ 334-338 (1989).

79. Ingham, C. A., Hood, S. H., Van Maldegem, B., Weenink, A. \& Arbuthnott, G. W. Morphological changes in the rat neostriatum after unilateral 6-hydroxydopamine injections into the nigrostriatal pathway. Exp. Brain Res. 93, 17-27 (1993).

80. Meredith, G. E., Ypma, P. \& Zahm, D. S. Effects of dopamine depletion on the morphology of medium spiny neurons in the shell and core of the rat nucleus accumbens. J. Neurosci. 15, 3808-3820 (1995).

81. Ingham, C. A., Hood, S. H., Taggart, P. \& Arbuthnott, G. W. Plasticity of synapses in the rat neostriatum after unilateral lesion of the nigrostriatal dopaminergic pathway. J. Neurosci. 18, 4732-4743 (1998)

82. Robinson, T. E. \& Kolb, B. Persistent structural modifications in nucleus accumbens and prefrontal cortex neurons produced by previous experience with amphetamin J. Neurosci. 17, 8491-8497 (1997).

83. Robinson, T. E. \& Kolb, B. Alterations in the morphology of dendrites and dendritic spines in the nucleus accumbens and prefrontal cortex following repeated treatment with amphetamine or cocaine. Eur. J. Neurosci. 11, 1598-1604 (1999).

These two papers show that the chronic in vivo administration of psychostimulants changes the morphology of dendritic spines in the nucleus accumbens and prefrontal cortex.

84. Hope, B. T. et al. Induction of a long-lasting AP-1 complex composed of altered Fos-like proteins in brain by chronic cocaine and other chronic treatments. Neuron 13 1235-1244 (1994).

85. Berke, J. D., Paletzki, R. F., Aronson, G. J., Hyman, S. E. \& Gerfen, C. R. A complex program of striatal gene expression induced by dopaminergic stimulation. J. Neurosci. 18, induced by dopam

86. Kuhar, M. J., Joyce, A. \& Dominguez, G. Genes in drug abuse. Drug Alcohol Depend. 62, 157-162 (2001).

87. Kelz, M. B. et al. Expression of the transcription factor $\triangle$ FosB in the brain controls sensitivity to cocaine. Nature 401, 272-276 (1999).

88. Bibb, J. A. et al. Effects of chronic exposure to cocaine are regulated by the neuronal protein Cdk5. Nature $\mathbf{4 1 0}$ 376-380 (2001).

89. Shaywitz, A. J. \& Greenberg, M. E. CREB: a stimulusinduced transcription factor activated by a diverse array of induced transcription factor activated by a diverse array of (1999).

90. Bourtchuladze, R. et al. Deficient long-term memory in mice with a targeted mutation of the CAMP-responsive elementbinding protein. Cell 79, 59-68 (1994).

91. Yin, J. C. et al. Induction of a dominant negative CREB transgene specifically blocks long-term memory in Drosophila. Cell 79, 49-58 (1994).

92. Nguyen, P. V., Abel, T. \& Kandel, E. R. Requirement of a critical period of transcription for induction of a late phase of LTP. Science 265, 1104-1107 (1994).

93. Frey, U., Frey, S., Schollmeier, F. \& Krug, M. Influence of actinomycin D, an RNA synthesis inhibitor, on long-term potentiation in rat hippocampal neurons in vivo and in vitro. J. Physiol. (Lond.) 490, 703-711 (1996).

94. Nguyen, P. V. \& Kandel, E. R. A macromolecular synthesisdependent late phase of long-term potentiation requiring
CAMP in the medial perforant pathway of rat hippocampal

95. Silva, A. J., Kogan, J. H., Frankland, P. W. \& Kida, S. CREB and memory. Annu. Rev. Neurosci. 21, 127-148 (1998)

96. Das, S., Grunert, M., Williams, L. \& Vincent, S. R. NMDA and $\mathrm{D} 1$ receptors regulate the phosphorylation of CREB and the induction of c-fos in striatal neurons in primary culture. Synapse 25, 227-233 (1997)

97. Konradi, C., Leveque, J. C. \& Hyman, S. E. Amphetamine and dopamine-induced immediate early gene expression in striatal neurons depends on postsynaptic NMDA receptors and calcium. J. Neurosci. 16, 4231-4239 (1996).

98. Cole, R. L., Konradi, C., Douglass, J. \& Hyman, S. E. Neuronal adaptation to amphetamine and dopamine: molecular mechanisms of prodynorphin gene regulation in rat striatum. Neuron 14, 813-823 (1995).

99. Hurd, Y. L. \& Herkenham, M. Molecular alterations in the neostriatum of human cocaine addicts. Synapse $\mathbf{1 3}$, 357-369 (1993).

100. Spanagel, R., Herz, A. \& Shippenberg, T. S. Opposing tonically active endogenous opioid systems modulate the mesolimbic dopaminergic pathway. Proc. Natl Acad. Sci. USA 89, 2046-2050 (1992).

101. Steiner, H. \& Gerfen, C. R. Dynorphin regulates D1 dopamine receptor-mediated responses in the striatum: relative contributions of pre- and postsynaptic mechanisms in dorsal and ventral striatum demonstrated by altered immediate-early gene induction. J. Comp Neurol. $\mathbf{3 7 6}$ 530-541 (1996).

102. Shippenberg, T. S., Bals-Kubik, R. \& Herz, A. Examination of the neurochemical substrates mediating the motivational effects of opioids: role of the mesolimbic dopamine system and D-1 vs. D-2 dopamine receptors. J. Pharmacol. Exp. Ther. 265, 53-59 (1993).

103. Shippenberg, T. S. \& Rea, W. Sensitization to the behaviora effects of cocaine: modulation by dynorphin and $\mathrm{k}$-opioid receptor agonists. Pharmacol. Biochem. Behav. 57, 449-455 (1997).

104. Carlezon, W. A. Jr et al. Regulation of cocaine reward by CREB. Science 282, 2272-2275 (1998)

105. Spangler, R. et al. Regulation of $\kappa$ opioid receptor mRNA in the rat brain by 'binge' pattern cocaine administration and correlation with preprodynorphin mRNA. Brain Res. Mol. Brain Res. 38, 71-76 (1996).

106. Cole, A. J., Bhat, R. V., Patt, C., Worley, P. F. \& Baraban, J. M. D1 dopamine receptor activation of multiple transcription factor genes in rat striatum. J. Neurochem. 58, 1420-1426 (1992).

107. Simpson, J. N., Wang, J. Q. \& McGinty, J. F. Repeated amphetamine administration induces a prolonged augmentation of phosphorylated cyclase response elementbinding protein and Fos-related antigen immunoreactivity in rat striatum. Neuroscience 69, 441-457 (1995).

108. Lyford, G. L. et al. Arc, a growth factor and activity-regulated gene, encodes a novel cytoskeleton-associated protein that is enriched in neuronal dendrites. Neuron 14, 433-445 (1995).

109. Cole, A. J., Saffen, D. W., Baraban, J. M. \& Worley, P. F. Rapid increase of an immediate early gene messenger RNA in hippocampal neurons by synaptic NMDA receptor activation. Nature $\mathbf{3 4 0}$, 474-476 (1989).

110. O'Brien, R. J. et al. Synaptic clustering of AMPA receptors by the extracellular immediate-early gene product Narp. Neuron 23, 309-323 (1999).

111. Yamagata, K. et al. Egr3/Pilot, a zinc finger transcription factor, is rapidly regulated by activity in brain neurons and colocalizes with Egr1/zif268. Learn. Mem. 1, 140-152 (1994).

112. Berridge, K. C. \& Robinson, T. E. What is the role of dopamine in reward: hedonic impact, reward learning, or incentive salience? Brain Res. Brain Res. Rev. 28, 309-369 (1998).

\section{(1) Online links}

\section{DATABASES}

The following terms in this article are linked online to:

LocusLink: http://www.ncbi.nlm.nih.gov/LocusLink/ adenylyl cyclase | AMPAR | Arc | calmodulin | CaMKIV | CBP | Cdk5 | CREB | D1 receptor | D2 receptor | dynorphin | ELK1 | Fos | $\Delta$ FosB | GluR2 | Homer | Jun | MKP-1 | Narp | NMDAR | א-opioid receptor | PKA | POLR2 | RSK | SRF | TBP MIT Encyclopedia of Cognitive Sciences: http://cognet.mit.edu/MITECS/

magnetic resonance imaging | positron emission tomography 